\title{
Surgical competence, research and evidence-based medicine (EBM) in orthopaedic surgery: what the ESSKA is doing to bring it all together
}

\author{
Laura de Girolamo $^{1} \cdot$ David Dejour $^{2} \cdot$ Martin Lind $^{3} \cdot$ Jon Karlsson ${ }^{4} \cdot$ Romain Seil $^{5}$ (I)
}

Received: 12 November 2019 / Accepted: 16 December 2019 / Published online: 23 December 2019

c European Society of Sports Traumatology, Knee Surgery, Arthroscopy (ESSKA) 2019

Keywords Surgeon-scientist $\cdot$ Evidence-based medicine $\cdot$ Career track $\cdot$ Research $\cdot$ Knowledge $\cdot$ Scientific society

The advancement of life sciences relies on educating new generations of professionals who, through their research efforts, can eventually improve patient care and thereby the quality of life of patients. In the fields of orthopaedic sports medicine and joint surgery, surgical innovation has been outstanding over the past few decades. Never before have so many patients been surgically treated at such a high level as today. Many surgeon investigators and orthopaedic pioneers have clearly changed the way that surgery is indicated and practised today, impacting global patient care through their innovative minds and their actions, while teaching future generations of surgeons, with the support of the academic world and our scientific societies.

However, this fragile ecosystem which is allowing the continuous generation and adoption of new knowledge into routine clinical practice is being threatened. In Europe, subspecialities like orthopaedic sports medicine are disappearing from academic centres and, more generally, there is a risk of facing the "disacademisation" of our scientific community. For many reasons, it is often difficult for surgeons

Romain Seil

rseil@yahoo.com

1 Orthopedic Biotechnology Laboratory, IRCCS Orthopedic Institute Galeazzi, Milan, Italy

2 Department of Knee Surgery, Lyon Ortho Clinic, Lyon, France

3 Department of Orthopaedics, Aarhus University Hospital, Aarhus N, Denmark

4 Department of Orthopaedics, Sahlgrenska University Hospital, Sahlgrenska Academy, University of Gothenburg, Gothenburg, Sweden

5 Department of Orthopaedic Surgery, Clinique d'Eich-Centre Hospitalier de Luxembourg, 78, rue d' Eich, 1460 Luxembourg, Luxembourg to acquire competence in science and research. There is a lack of harmonisation and a structural gap in acquiring research competence during and after medical studies in Europe. Likewise, residency training often focuses essentially on clinical competence and has no research component [3]. Legal regulations like the EU working time directive may add an additional limit to the time available for surgical education and scientific work during residency training. Finally, the increasing economic pressure and need for clinical productivity in current healthcare systems are leading to surgeons frequently only being seen as technicians who need to operate to ensure a healthy bottom line for their institution [1].

The ESSKA's involvement in this changing environment can be seen at different levels. A set of core competencies has been created for ESSKA specialists, known as the core curriculum. Despite the geographical differences that characterise Europe, ESSKA members have agreed on the surgical competencies that should be mandatory for a young orthopaedic surgeon specialising in sports traumatology and sports medicine and also for the experienced surgeon subspecialising in complex surgery. This upcoming core curriculum could serve as a guideline for educational direction in sports traumatology, sports medicine and knee surgery. The recently launched series of 'All About...' courses aims to give the participants specific knowledge within a given field and, if a participant completes the full set of coursesknee, shoulder, ankle, hip, elbow and forearm, he/she will have reached a required standard, a sort of unofficial certification at European level. The technical aspect, as provided by the cadaver courses, is accompanied by the theoretical basis, resulting in efficient, practical certification that would guarantee full quality of treatment. These courses and more generally the core curriculum project are both designed for young surgeons, so that they can direct their career from 
the start, and to update the most experienced ones as well. Although not fully sufficient to support a surgeon-scientist development, the core-curriculum programme is a very useful tool, especially for the "young thrusters", who are taking their first steps towards such a career.

In fact, competence in science and research is not systematically acquired during medical studies or subsequent medical specialisation. It can be divided into three categories [3]. Generic competence includes the ability to synthesise findings and draw conclusions from these findings. That related to "using research" contains the ability to define and carry out an appropriate literature search and to appraise research evidence critically. Finally, the competence related to 'doing research' constitutes the ability to formulate a research question as a hypothesis and to analyse data. While acquiring this competence during medical studies or later during residency training is considered to be important across Europe, opportunities to learn about research in medical or postgraduate training in Europe vary enormously [3]. Surgeons should therefore not only be able to use research, but also to conduct research.

When people hear the word "research", the first thought that immediately comes into their minds is a laboratory scientist working at a bench surrounded by strange chemical compounds and observing cells under a microscope. On the other hand, when people hear the expression "patient care", their first thought is a clinician in a white coat next to the patient's bed or busy with complex surgical procedures. However, there's a "trait d'union" between these two worlds which are apparently very distant but are actually strictly connected, namely the clinical scientists. They are the unique, precious and mandatory interface between laboratory science and frontline clinical practice in supporting clinical partnerships for evidence-based good clinical practice, stimulating medical innovations through more strict interactions with emerging fields.

As a leading scientific society in the field of orthopaedics and sport medicine in Europe, the ESSKA recognises that surgical research has revolutionised and transformed the treatment of many complex diseases and identifies the need for a larger number of surgeon-scientists in the orthopaedic and sports medicine community. Career paths for surgeonscientists have always been arduous, but the current healthcare environment has made this career almost prohibitively challenging [2]. Analysing the career track in surgery, it is possible to identify a dual pathway, namely clinical and scientific. In science, third-cycle scientific careers usually start after a candidate has earned a bachelor's and a master's degree and end with a Ph.D. graduation. In medicine, this process generally starts 5-7 years later (Fig. 1), provided that a medical student shows an interest in scientific studies, which is the case in a minority. In surgery, the delay can be further explained by the need to acquire surgical expertise - which is made up of both theoretical knowledge and practical skills - before treating patients at a high level and being able to generate new surgery-related knowledge and innovations. Research at surgical departments is not always clearly encouraged, whereas it is in contrast highly valued for the non-surgical specialities and basic science departments. The issue is exemplified by the fact that nonsurgeon physicians apply 2.5 times more than surgeons for any type of National Institutes of Health (NIH) career development award [4]. Consequently, while the figure of the physician-scientist is well represented, that of the surgeonscientist is far rarer.

In this scenario, the ESSKA embraces the current changes in the surgical environment by defining the competence needed for individuals to become proficient in orthopaedic sports medicine and joint surgery, structuring the related practical skills and theoretical knowledge and stimulating science and research to improve patient care and thereby the quality of life of patients, according to its mission statement.

For most orthopaedic surgeons, irrespective of their research career, the challenge is to keep up with the pace of the generated new knowledge and to differentiate between good and evil evolutions. So many channels of information are available through the social media that being critical of "biased" information and data remains difficult. Ideally, their practice should rely on principles which are backed up by evidence-based medicine. EBM, defined by Sackett in 1996 as "the conscientious, explicit, and judicious use of current best evidence in making decisions about the care of individual patients" [5], should be a firm reference point along the entire career path of a surgeon. EBM that integrates medical knowledge and scientific evidence with patients' needs and preferences has had a considerable impact on all fields of primary care, including medicine, nursing, physiotherapy, occupational therapy, nutrition and dentistry, as well as the area of healthcare management and economics [6]. Nevertheless, the awareness of EBM among the community of ESSKA surgeons and its application in daily clinical practice are not so obvious. Some peculiar and interesting findings emerged in the original paper by Lugano et al., published in this issue ([page XXX). The aim of this cross-sectional survey was to investigate the awareness, knowledge and perception of EBM amongst orthopaedic surgeons and healthcare specialists in the ESSKA. The survey identified some interesting findings in the application of EBM principles. In addition to showing some geographical differences, it revealed that the application of EBM is more difficult for the youngest surgeons, that it is positively affected by the time dedicated to research and that it is negatively correlated with the time dedicated to patient care. Despite the medical community's knowledge of and interest in EBM, these results highlight the need for further investigation into the main reasons behind the limited diffusion of the EBM approach. 

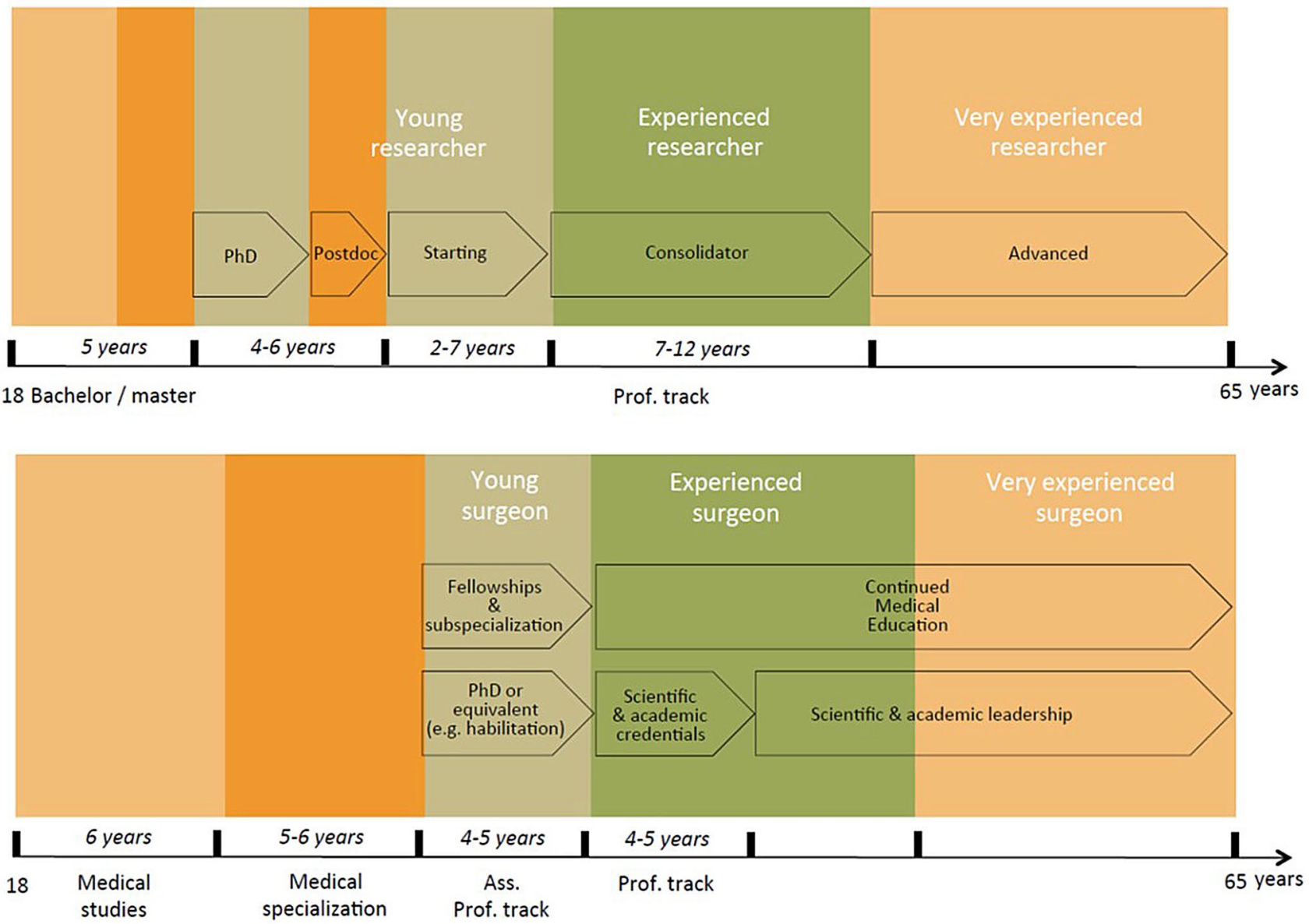

Fig. 1 Difference in years between scientific tracks (top) and surgeon-scientist tracks (bottom)

Properly applied, EBM has the potential to upgrade clinical practice, linking medical knowledge and scientific evidence to patients' needs, eventually leading to undoubted benefits to patients and more generally to the healthcare system. Current evidence suggests that clinically integrated EBM training is effective and tailored e-learning packages and evidence-based journal clubs might be a potential strategy for improving the knowledge and skills of EBM for surgeons of the next generation. In this respect, active scientific societies such as the ESSKA must play a fundamental role in supporting the knowledge of EBM and its application in daily clinical practice. The first step in this direction has been made by the ESSKA through the recent implementation of its first 'all about clinical studies and publishing course', as well as its huge efforts to promote EBM through its scientific journals, the KSSTA and JEO, and through the ESSKA Academy, an e-learning portal and educational platform which presents all the current trends and guidelines in an easy format.

The role of scientific societies like the ESSKA is to build and disseminate scientific knowledge and scientific progress, enhance educational interaction, stimulate creative thinking and learning, exchange ideas, data and content, promote research and foster scientific discussion and publication. The ESSKA provides its members with access to a community of peers from whom they can both learn and seek feedback on their own work. It offers networking opportunities to younger colleagues for academic advancement. Furthermore, surgical scientific societies need to disseminate practical knowledge to allow surgeons to perform their procedures with proficiency. They must also contribute to the development of new and innovative surgical products and instruments to make surgery safer and less invasive for patients. The role of our scientific society is always to be critical and encourage surgeons to look at the results and scientific content to validate the "new appealing trends".

The ESSKA's recent achievements, like the development of a core curriculum and the creation of its 'ALL ABOUT...' series, are a testimony to the ESSKA's dynamism and its desire continuously to adapt to its changing environment. In this way, it fulfils its catalytic function in an ever-increasing competitive academic environment, builds on European traditions and contributes to the development of surgical science. 
Authors contribution All the authors contributed to the preparation of the Editorial note.

Funding No funding has been received.

\section{Compliance with ethical standards}

Conflict of interest The authors declare no conflict of interest regarding the present work.

Ethical approval The Editorial note needs neither ethical approval nor informed consent.

\section{References}

1. Evers BM (2015) The evolving role of the surgeon scientist. J Am Coll Surg 220:387-395
2. Mann M, Tendulkar A, Birger N, Howard C, Ratcliffe MB (2008) National Institutes of Health funding for surgical research. Ann Surg 247:217-221

3. Marz R, Dekker FW, Van Schravendijk C, O'Flynn S, Ross MT (2013) Tuning research competences for Bologna three cycles in medicine: report of a MEDINE2 European consensus survey. Perspect Med Educ 2:181-195

4. Rangel SJ, Moss RL (2004) Recent trends in the funding and utilization of NIH career development awards by surgical faculty. Surgery 136:232-239

5. Sackett DL, Rosenberg WM, Gray JA, Haynes RB, Richardson WS (1996) Evidence based medicine: what it is and what it isn't. BMJ 312:71-72

6. Slowther A, Ford S, Schofield T (2004) Ethics of evidence based medicine in the primary care setting. J Med Ethics 30:151-155

Publisher's Note Springer Nature remains neutral with regard to jurisdictional claims in published maps and institutional affiliations. 